\title{
Derivation of non-classical stochastic price dynamics equations
}

\author{
Carey Caginalp ${ }^{1,2}$ and Gunduz Caginalp ${ }^{1}$
}

July 20, 20202 pm

${ }^{1}$ Mathematics Department, University of Pittsburgh, Pittsburgh, PA 15260

${ }^{2}$ Economic Science Institute, Chapman University, Orange, CA 92866

E-mail: CC carey_caginalp@alumni.brown.edu, GC caginalp@pitt.edu

Keywords: asset price dynamics, fat tails, stochastic differential equations, supply/demand

Abstract. We analyze the relative price change of assets starting from basic supply/demand considerations subject to arbitrary motivations. The resulting stochastic differential equation has coefficients that are functions of supply and demand. We derive these rigorously. The variance in the relative price change is then also dependent on the supply and demand, and is closely connected to the expected return. An important consequence for risk assessment and options pricing is the implication that variance is highest when the magnitude of price change is greatest, and lowest near market extrema. This occurs even if supply and demand are not dependent on price trend. The stochastic equation differs from the standard equation in mathematical finance in which the expected return and variance are decoupled. The methodology has implications for the basic framework for risk assessment, suggesting that volatility should be measured in the context of regimes of price change. The model we propose shows how investors are often misled by the apparent calm of markets near a market peak. Risk assessment methods utilizing volatility can be improved using this formulation.

Keywords: asset prices, stochastic models, price variance, risk, volatility, supply and demand.

JEL Classification: C00, G12, G40 
1. Introduction. While equilibrium price theory has been developed extensively in classical economics, the study of dynamics that describes the path to equilibrium is still in the developing stage. Broadly speaking, the approaches can be divided into (i) discrete models - based on an imbalance of supply and demand - that have typically been considered for goods and commodities, (ii) continuum models - often incorporating randomness - for asset price dynamics that are standard in options pricing and risk assessment.

1.1. A simple and standard model of type (i) above is the excess demand model, which is often stated in classical economics 1 as

$$
p_{t}-p_{t-1}=\frac{1}{\tau_{0}}\left(d_{t-1}-s_{t-1}\right)
$$

where $p_{t}$ is the price at the discrete time, $t$, and $s_{t-1}$ and $d_{t-1}$ are the supply and demand at time $t-1$, and $\tau_{0}^{-1}$ is a constant that determines the extent to which prices move for each unit of imbalance between supply and demand (e.g. Watson and Getz, 1981 [34] or Weintraub 1979 [35]). This is obtained directly from supply and demand functions that are assumed to be straight lines, which are good approximations for small deviations when these functions are smooth. Of course, (11) is only a local equation that is valid for a particular pair of linear supply and demand. For example, an imbalance created by $d_{t-1}=10,020$ and $s_{t-1}=10,000$ will have a much smaller impact on price change than would $d_{t-1}=40$ and $s_{t-1}=20$. This demonstrates the need for normalization, realized by dividing the right hand side of (10) by $s_{t-1}$. Similarly, the left hand side must be normalized by dividing by $p_{t-1}$ leading to the equation

$$
\frac{p_{t}-p_{t-1}}{p_{t-1}}=\frac{1}{\tau_{0}} \frac{d_{t-1}-s_{t-1}}{s_{t-1}}
$$

While these normalizations lead to an equation that is a reasonable non-local model, another feature of (1) is that it is a linear equation, so that the price change is always proportional to the excess demand. While linearity is often a convenient and reasonable approximation, there is no compelling requirement that price change be a linear function of excess demand. Introducing a differentiable function $g: \mathbb{R}^{+} \rightarrow \mathbb{R}$ with suitable properties including $g(1)=0$ and $g^{\prime}>0$, we can write in place of (1) the equation

$$
\tau_{0} \frac{p_{t}-p_{t-1}}{p_{t-1}}=g\left(\frac{d_{t-1}}{s_{t-1}}\right) \text {. }
$$

Thus, information on the supply and demand at a any discrete time determines the price change for the next discrete time. The design of markets and

\footnotetext{
${ }^{1}$ Walrasian equilibrium is defined by zero excess demand, $E=D-S$, i.e., the right hand side of (1), vanishes so that the price is unchanged in the next time period. The price thereby adjusts to the quantity. A tatonnement process (see e.g., 35]), such as (1) is one in which trading occurs only at equilibrium. When trading at the discrete time $t$ has ended, the intersection of the new supply and demand then determines the price $P_{t+1}$ of the next trade.
} 
efficient price discovery has been an active research area from both a theoretical and experimental perspective. See models in Milgrom, 2017 [26] and Gjerstad and Dickhaut, 1998 [16], Hirshlefer et. al., 2005 [20], Gjerstad, 2007 [17] and 2013 [18] and references therein. The experimental aspect has been studied by researchers including Plott and Pogorelskiy, 2017 [27, Crocket et. al., 2009 [16], Bossaerts and Plott, 2004 [4, Porter and Rassenti, 2003 [28.

1.2. At the continuum level, the focus of research for several decades has been on the price dynamics of asset prices, such as stocks and options, subject to randomness. A standard equation that has been the starting point for much of mathematical finance is written in terms of Brownian motion $W(t ; \omega)$, with $\omega \in \Omega$, the probability state space, as

$$
P^{-1} \frac{d P}{d t}=\mu d t+\sigma d W .
$$

Here $P$ is the price as a function of continuous time, $t$, while $\mu$ and $\sigma$ are the expected return and standard deviation. The parameter $\mu$ is the expected return (often based on historical data), and $\sigma$ is often set based on the idea (that has some limited empirical justification) that volatility remains relatively stable in time. These parameters are often assumed to be constant, and in some cases a prescribed function of time. This equation has a long history, with the main ideas dating back to Bachelier, 1900 [1] and now utilized in basic textbooks such as Karatzas and Shreve, 1998 [22] and Wilmott, 2013 [36. With no information besides some price history, one can regard (4) as a good first approximation for several mathematical problems such as options pricing and risk assessment.

The approach leading to (4) does not build on the basic economic ideas of supply and demand, but rather has limited justification based on empirical observation and is appealing due to the salient mathematical properties. One of the deficiencies of (4) is that it vastly understates the risk for unusual events. For example, if one calculates the probability of, say, a $4 \%$ or $5 \%$ drop in a stock index based upon the empirical daily standard deviation, one obtains a result that is vastly smaller than empirical observations. While many explanations (with some justification) have been offered for this anomaly, often called "fat tails," Caginalp and Caginalp 2018, 7, 2019, 8] showed that it arises as a consequence of the mathematical property that a quotient of normals is approximately normal in part of the domain of the density (see Díaz-Francés and Rubio 2013 [13], Champagnat et. al. 2013 [10] and references therein), but not near the tail, where it can be a power law rather than exponential. The basic idea is that supply and demand can be expected to be normal as a consequence of the Central Limit Theorem. Thus, their quotient as one has in (22) or (3) will not be close to normal near the tail of the distribution, i.e., for rare events.

A practical examination of the source of randomness in asset prices shows that the overwhelming fraction of the randomness arises from the randomness in supply and demand. In other words, if one knew how the supply and demand would change at the next time interval, one would essentially know how prices 
would evolve2. In trading of a major stock or index, for example, there are dozens of professionals focusing on the same stock and whose sole business consists of exploiting very small deviations from optimal pricing. Indeed, these professionals observe the same shifts in supply and demand again and again, and earn their living by adjusting their bids and asks in response to these shifts.

These professionals, some of whom are "market makers" tasked with ensuring orderly markets, are not concerned with the fundamentals or even the long term trends. At any given moment they observe the change in the supply demand curves regardless of origin of those changes. Given the change in supply and demand, there will be a unique price that is clearly based on previous iterations. If some of the professionals have biases or irrational expectations whereby they are not able to deduce the correct new price, they will not be in business very long since there are many trades throughout one day, so that a losing strategy will exhaust the capital in a short time. This does not contradict the premises of behavioral finance since there are many aspects of trading, with some having an efficiency higher than others. The perspective of the market makers is that they have some capital for their business which involves adjusting to the orders of a stock. Unlike individual or hedge fund investors or even mutual fund managers, the market makers cannot tie up their business capital based on their assessment of either the fundamental value or the long term trend (and other technical indicators), or other beliefs they may hold about the stock.

The situation is illustrated in Figure 1. The supply-demand graph displays the initial supply function (which is increasing) in solid red and the (decreasing) demand function in solid blue. The price is established by the intersection at the price $P=1.29$. A short time later, there is a random event (e.g., a news announcement such as an earnings report) that changes the supply and demand for the asset. In particular, if the news is positive, it will increase the demand, so the solid blue curve shifts upward to the dotted blue line. The potential sellers are also aware of the positive news and re-adjust their orders to reflect the changing circumstances, thereby raising the price that they are willing to accept. The solid red curve thus shifts upward to the dotted red curve. The new supply and demand functions now intersect at a higher price, $P=1.44$. The key point is that the random event influences a large number of agents in terms of their preferences to buy or sell, so that the impact of the randomness is entirely comprised of the shifts in the supply and demand curves. Given the shifts in the supply and demand curves, there is little additional source of randomness given the large number of market makers who are seeking to capitalize on these shifts and have optimized on the same shift many times.

\footnotetext{
${ }^{2}$ One can almost regard this statement as a tautology since the price is determined exclusively by the supply and demand functions. Theoretically, the price change after a small time increment will be determined by the change in these functions. In many markets there are features that complicate the supply/demand analysis, such as market makers who can buy on their own account at better prices than the traders.
} 


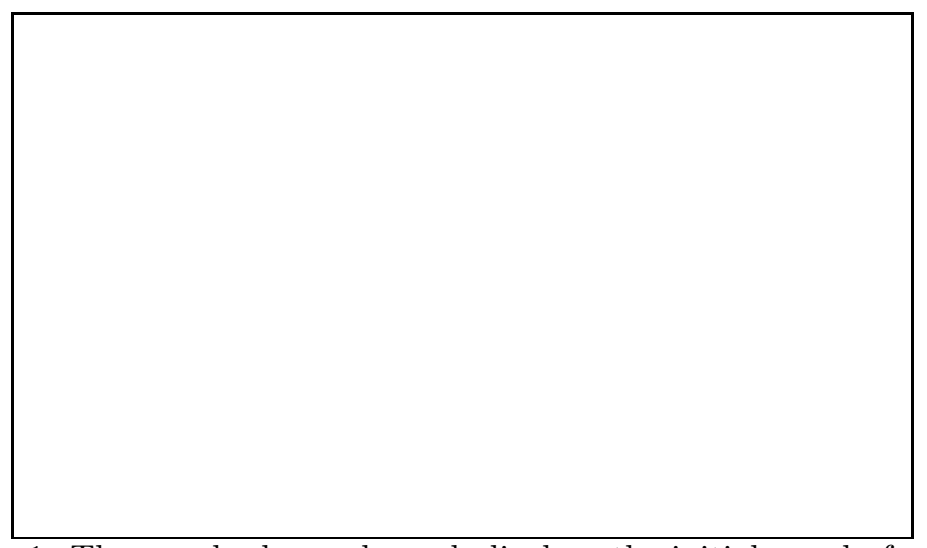

Figure 1. The supply-demand graph displays the initial supply function (which is increasing) in solid red and the (decreasing) demand function in solid blue. The price is established by the intersection at the price $P=1.29$. A short time later a random event increases demand and decreases supppy, so the blue curve shifts upward to the dotted blue line, and the supply shifts downward to the dotted red line, establishing a higher price, $P=1.44$.

Examples of company specific news that influences the supply/demand curves include quarterly revenue and earnings reports, changes in the company's leadership, securing a lucrative contract, announcements on restating previous earnings, government announcements of investigations into the company, etc. For the broader market, the supply/demand curves often shift with government updated indicators, most of them monthly, such as the nonfarm payrolls, stating the net number of jobs added or lost during the preceding month, the retail sales changes, the consumer price increases, trade balance numbers, etc. Other factors are also include changes in interest rates, i.e., the bond markets, international trade and currencies, natural disasters, etc. Large changes in supply/demand occur when there is large deviation from the expected outcome. For example, during both the financial crisis of 2008 and the Covid pandemic of 2020, the employment announcements were very significant as the markets braced for job loss of hundreds of thousands or millions, respectively. What is often surprising to non-experts, however, is that supply/demand curves prior to an announcement are based on the current forecast. If that forecast is for two million jobs lost, and the news is that "only" one million jobs are lost, this is favorable news, and the supply (of shares submitted for sale in the S\&P index, for example) often shifts down, and the demand shifts up (as displayed in Figure 1). Thus, the impact of the news is always relative to the existing expectations.

This idea can be tested empirically in exchanges and experimentally in laboratories. Given the same shift in supply and demand how much variance will there be in a market with many experienced market makers and short term traders? We claim that it will be negligible compared to the randomness in supply and demand arising from news items (e.g., earnings reports, forecasts 
and analysts reports for a stock) and influx or outflow of funds for a particular asset.

In particular, one can design a market in which news (altering the payout) will arrive at various times. In addition to the usual traders, there will be short term traders who have the constraint that they must have zero inventory of the asset at the end of each period (similar to a market maker). By analyzing the supply/demand changes throughout the experiment, one can determine the variance in the relative price change among the times when the shift in supply/demand is nearly identical. The hypothesis is that when the shift in supply/demand is similar, so is the price change. On the other hand, one can determine the variance in the supply/demand shift given a spectrum of news that impacts the asset payoff.

Thus, a fundamental analysis of randomness in asset prices should begin with an examination of the process by which randomness in supply and demand propagates to the stochastics of price change.

Our goal here is to present a precise derivation and justification of an equation analogous to (4) that is based on supply and demand considerations (see Caginalp and Caginalp, 2019, [9]), namely,

$$
P^{-1} d P=G(D / S) d t+\sigma \frac{D}{S} G^{\prime}(D / S) d W
$$

Here, $S$ and $D$ be the expected value of supply and demand, respectively. The basic premise is that the relative change in price, $P^{-1} d P / d t$ in terms of a function $G$, which is analogous to $g$ above, and meets the requirements specified in Section 2. Since $G$ depends on the ratio of total supply, $\tilde{S}$, and demand, $\tilde{D}$, one has the basic equation $P^{-1} d P / d t=G(\tilde{D} / \tilde{S})$. Writing

$$
\tilde{D}(t, \omega)=D(t)\left(1+\frac{\sigma}{2} R\right), \quad \tilde{S}(t, \omega)=S(t)\left(1-\frac{\sigma}{2} R\right)
$$

with $R$ as the standard normal, denoted $\mathcal{N}(0,1)$, and expanding $G$ in a Taylor series formally leads to (5) in the limit (see 9 for details). One important consequence of this equation is that the volatility (as defined in Section 5) will be a function of $D / S$, as is the price derivative. Prior research [9] concludes that volatility will be a minimum at price extrema, and a maximum when the ratio $D / S$ (and consequently the magnitude of the price derivative) are at a maximum. Of course, for market bottoms, there may be other factors at work, e.g., margin calls, that would need to be modeled, as discussed in Section 5. Equation (5) has important implications for risk assessment and options pricing. For example, if one is using the classical equation (41), and measuring $\sigma$, the risk and volatility would be underestimated if the trading price of the asset is near a market top. There is empirical evidence that major stock market tops are associated with low volatility (Sornette et. al., 2018 [31]) suggestive of 
the maxim "calm before the storm." The supply, $S(t)$ and demand, $D(t)$, in (5) can be specified, or be coupled to other differential equations, such as the asset flow equations that have been developed since the late 1980's (see e.g., Caginalp and Balenovich, 1999 [6], Merdan and Alisen, 2011 [25], DeSantis and Swigon, 2018 [12], and references therein).

While volatility is an active research area (see e.g., [15, [21, 30, 32]) and advances in empirical calculations of volatility have been made in recent years, an approach based on microeconomics and (5) can be instrumental in an integrated understanding of price change and volatility.

In this paper, we present a rigorous derivation of the probability density corresponding to (5). This provides a justification of the fat tail properties established in 7 that demonstrated that a power law decay in relative price change is a consequence of the probability distribution in inherent in the supply/demand ratio. In other words, assuming that supply and demand are normally distributed (as one would expect from the Central Limit Theorem) the quotient will have a density that decays (in many cases) as a power law. Furthermore, we use this density to derive the stochastic equation (5).

2. Derivation of the density for stochastic asset dynamics equation. In this section we derive rigorously the density of the relative price change within $(t, t+\Delta t)$ that is generated by (5). The exact result is given by (18) below.

First, we define the requirements for a function $G: \mathbb{R}^{+} \rightarrow \mathbb{R}$ that will specify the nature of the relative price change as a function of the ratio of demand to supply. In order to be compatible with basic ideas of economics, $G$ must be increasing (see (ii) below). For $S$ and $D$ to be on an equal footing, one needs the condition (iii) below. The other conditions have been discussed in [7].

2.1 Condition $G$. The function $G: \mathbb{R}^{+} \rightarrow \mathbb{R}$ is a twice continuously differentiable function satisfying

(i) $G(1)=0,($ ii $) G^{\prime}(x)>0$ all $x \in \mathbb{R}^{+}$, (iii) $G(x)=-G\left(\frac{1}{x}\right)$,

$$
\begin{gathered}
\text { (iv) } \lim _{x \rightarrow \infty} x G^{\prime}(x)=\infty \text { and } \lim _{x \rightarrow 0+} x G^{\prime}(x)=\infty . \\
\text { (v) }\left(x G^{\prime}(x)\right)^{\prime} \quad \text { is }\left\{\begin{array}{lll}
<0 & \text { if } & x<1 \\
>0 & \text { if } & x>1
\end{array} . / / /\right.
\end{gathered}
$$

These properties clearly imply the following two relations:

$$
\begin{gathered}
x G^{\prime}(x)=\frac{1}{x} G^{\prime}\left(\frac{1}{x}\right) . \\
\lim _{x \rightarrow \infty} G(x)=\infty .
\end{gathered}
$$

In deriving the stochastic equations for price change, additional conditions are needed, and listed in the augmented condition below. 
Condition $G_{A}$. Let $G$ satisfy Condition $G$, and, in addition, assume that $G^{-1}$ has four continuous derivatives that are bounded on bounded subsets of the domain.

\subsection{Examples of functions that satisfy Condition $G_{A}$.}

One can readily verify that the following functions satisfy this condition:

(i) $G(x)=x^{q}-x^{-q}$ for $q>0$;

(ii) $G(x)=\left(x-x^{-1}\right)^{q}$ for $q$ an odd positive integer.

Note that when $x-1=\frac{D-S}{S}$ is small, the basic model $(i)$ with $q:=1$ is the continuous analog of the simple excess demand model often considered in economics, i.e., $g\left(\frac{D}{S}\right)=\frac{D-S}{S}$, in (3)).

2.3. The derivation. We denote by $\tilde{D}$ the total demand including randomness per unit time. During a time interval $(t, t+\Delta t)$, the total demand is $\tilde{D} \Delta t$ while the deterministic component is $D \Delta t$, where $D$ (which can depend on time, price and other factors) can be regarded as the expected value of $\tilde{D}$. In the same way, the supply $\tilde{S}$ and expectation of supply, $S$ are defined.

We can then write the total demand during time $\Delta t$ divided by the total supply in that time period as

$$
\frac{\tilde{D} \Delta t}{\tilde{S} \Delta t} .
$$

We argue below that given a large number of decision makers, the Central Limit Theorem states, under broad conditions, that the randomness in the buy orders placed by many different agents will be approximately Gaussian (as discussed further in Section 2.3). Note that this is a very different - and more easily justified - assumption than the hypothesis that stock prices changes are lognormal. There is no convincing argument that asset price changes should satisfy the hypotheses of the Central Limit Theorem, since price adjustments evolve in a complex manner through the supply and demand.

This does not mean that the supply and demand will be centered around values that would be consistent with fundamental value. For example, if there is a very positive image portrayed about some company, we still expect a normal distribution in the demand as a function of the price that the potential buyers are willing to pay, though that price may be much higher than would be indicated by finance value calculations such as the potential dividend stream. Also, there are, of course, correlations among groups of investors. However, for actively traded stocks, there are such a large number of such groups (some focusing on trend, others on fundamentals, etc.) which act independently that these correlations would cease to be relevant. Casual observations indicate that supply and demand, like may other preference issues, have a distribution that is qualitatively similar to a Gaussian, except near the tail, which is usually irrelevant since it is far from the crossing of supply and demand. While many studies have been performed on the distribution of relative price changes, more study is needed for the distribution of supply and demand distribution. Tests 
on their normality are the ultimate criterion for determining the validity of the assumption on an empirical basis.

We consider first the impact of randomness on supply and demand. As discussed in Appendix A, it is the "market orders" rather than the "limit orders" that are mainly responsible for changes in price. Since there are many independent agents using publicly available information to make decisions on trading a particular asset, we can assume that the orders are independent and identically distributed with a given mean and variance. During this time interval there will be additional demand (positive or negative) due to randomness from a variety of sources such as news items that alter the value or desirability of the asset. For example, if there is an unexpected announcement that the Federal Reserve is lowering interest rates, it may increase the demand for a stock index by $1 \%$. In other words, the random term in the demand is proportional to the baseline deterministic demand (i.e., the expected demand during this time interval, $D \Delta t$ ). Denoting by $\frac{\sigma}{2} R$ this random term per unit demand and per unit time, one has then that the total random factor in demand is given by $\frac{\sigma}{2} D R \Delta t$ and the total demand, $\tilde{D} \Delta t$, and supply, $\tilde{S} \Delta t$, during the time interval $(t, t+\Delta t)$ is given by

$$
\tilde{D} \Delta t=D \Delta t\left(1+\frac{\sigma}{2} R\right), \quad \tilde{S} \Delta t=S \Delta t\left(1-\frac{\sigma}{2} R\right) .
$$

The simple assumption is that a random event that increases demand for the asset decreases supply. This assumption can be relaxed as discussed below so that any correlation between the two random variables (for supply and demand, respectively) can be considered.

The random term, $\frac{\sigma}{2} R \Delta t$, is actually the average of a large number of agents with a distribution that we cannot necessarily specify. However, the Central Limit Theorem indicates that the limiting distribution will be normal since a sufficiently large number of them, having the same public information, can be assumed to have a particular distribution. One can then expect that $R$ will be a standard normal, often expressed as $\mathcal{N}(0,1)$, denoting a Gaussian with mean 0 and variance 1 , so that the term $D \Delta t \frac{\sigma}{2} R$ is normal with mean zero and variance $\left(D \Delta t \frac{\sigma}{2}\right)^{2}$.

If we accept the standard assumptions (see Schuss, 2009 29], p. 39) of the independence of the random events on $(t, t+\Delta t)$ from events prior to $t$, as well as the appropriate continuity properties in the limit as $\Delta t \rightarrow 0$, then we can assume that $R \Delta t=\Delta W:=W(t+\Delta t)-W(t)$. Summarizing, we write the total demand and supply on this interval as

$$
\tilde{D} \Delta t=D \Delta t+\frac{\sigma}{2} D \Delta W, \quad \tilde{S} \Delta t=S \Delta t-\frac{\sigma}{2} S \Delta W .
$$

Given, as noted above, that $R$ can be interpreted as the fractional random component per unit time. This is consistent with the interpretation of $R$ as $\Delta W / \Delta t$ which, in the limit as $\Delta t \rightarrow 0$, is "white noise" or the derivative of Brownian motion, which does not exist in the classical mathematical sense. Nevertheless, we do not need to utilize the limit of $\Delta W / \Delta t$ concept here. 
The relative change in price $P^{-1} d P / d t$, in the time interval $\Delta t$, is then postulated to be proportional to a function, $G$ of the total demand divided by supply during this time interval, i.e.,

$$
P^{-1} \frac{\Delta P}{\Delta t}=G\left(\frac{D \Delta t\left(1+\frac{\sigma}{2} R\right)}{S \Delta t\left(1-\frac{\sigma}{2} R\right)}\right) .
$$

We assume that $G$ satisfies condition $G_{A}$ and incorporates the time constant.

Using the standard normal random variable, $Y \sim \mathcal{N}(0,1)$ this equation has the form

$$
\begin{aligned}
P^{-1} \frac{\Delta P}{\Delta t} & =G\left(\frac{D}{S} \frac{\Delta t+\frac{\sigma}{2} Y(\Delta t)^{1 / 2}}{\Delta t-\frac{\sigma}{2} Y(\Delta t)^{1 / 2}}\right) \\
& \sim G\left(\frac{\mathcal{N}\left(D \Delta t, D^{2} \frac{\sigma^{2}}{4} \Delta t\right)}{\mathcal{N}\left(S \Delta t, S^{2} \frac{\sigma^{2}}{4} \Delta t\right)}\right)
\end{aligned}
$$

with numerator and denominator anti-correlated. The more general assumption that the supply and demand have a correlation different from -1 can be considered by assuming

$$
P^{-1} \frac{\Delta P}{\Delta t}=G\left(\frac{D}{S} \frac{\Delta t+\frac{\sigma}{2} Y_{1}(\Delta t)^{1 / 2}}{\Delta t+\frac{\sigma}{2} Y_{2}(\Delta t)^{1 / 2}}\right)
$$

where $Y_{1}$ and $Y_{2}$ are both standard normals, i.e., $\mathcal{N}(0,1)$, but have some correlation $\rho>-1$. The analysis is then more complicated, but the essential ideas are similar.

We proceed with the analysis of the basic equation (11) by defining random variables in order to obtain the density function for the random variable on the right hand side.

Toward this end, define

$$
\begin{aligned}
& X:=\frac{\Delta t+\frac{\sigma}{2} Y(\Delta t)^{1 / 2}}{\Delta t-\frac{\sigma}{2} Y(\Delta t)^{1 / 2}}, \quad X_{1}:=\frac{D}{S} \frac{\Delta t+\frac{\sigma}{2} Y(\Delta t)^{1 / 2}}{\Delta t-\frac{\sigma}{2} Y(\Delta t)^{1 / 2}} \\
& X_{2}:=G\left(\frac{D}{S} \frac{\Delta t+\frac{\sigma}{2} Y(\Delta t)^{1 / 2}}{\Delta t-\frac{\sigma}{2} Y(\Delta t)^{1 / 2}}\right), \quad X_{3}:=G\left(\frac{D}{S} \frac{\Delta t+\frac{\sigma}{2} Y(\Delta t)^{1 / 2}}{\Delta t-\frac{\sigma}{2} Y(\Delta t)^{1 / 2}}\right) \Delta t \\
& X_{1}=\frac{D}{S} X, \quad X_{2}=G\left(X_{1}\right), \quad X_{3}=G\left(X_{1}\right) \Delta t .
\end{aligned}
$$

The price equation can then be written as

$$
P^{-1} \Delta P=G\left(X_{1}\right) \Delta t=X_{3} .
$$


This means that the change in the relative price is governed by the random variable $X_{3}$, whose density we compute below, and show that it is approximately norma 3 for small $\sigma$ and fixed $\Delta t$. The first of the random variables above, namely $X$, is a quotient of two anticorrelated normal random variables for which one has an exact expression. In particular, Theorem 4.3 of Caginalp and Caginalp, 2018 [7] can be applied to $X$ in the form

$$
X=\frac{1+\frac{\sigma}{2(\Delta t)^{1 / 2}} Y}{1-\frac{\sigma}{2(\Delta t)^{1 / 2}} Y} .
$$

to yield the density of $X$ as

$$
f_{X}(x)=\frac{\frac{\sigma}{(\Delta t)^{1 / 2}}}{\sqrt{2 \pi}} \frac{e^{-\frac{1}{2} \frac{(x-1)^{2}}{\frac{1}{\Delta t}\left(\frac{\sigma}{2}\right)^{2}(x+1)^{2}}}}{\frac{1}{(\Delta t)}\left(\frac{\sigma}{2}\right)^{2}(x+1)^{2}} .
$$

The density of $X_{1}:=\frac{D}{S} X$ is then calculated as

$$
\begin{aligned}
f_{X_{1}}(x) & =\frac{f_{X}\left(\frac{x}{D / S}\right)}{D / S}=\frac{\frac{\sigma}{(\Delta t)^{1 / 2}}}{\sqrt{2 \pi} \frac{D}{S}} \frac{e^{-\frac{1}{2} \frac{\left(\frac{x}{D / S}-1\right)^{2}}{\frac{1}{\Delta t}\left(\frac{\sigma}{2}\right)^{2}\left(\frac{x}{D / S}+1\right)^{2}}}}{\frac{1}{\Delta t}\left(\frac{\sigma}{2}\right)^{2}\left(\frac{x}{D / S}+1\right)^{2}} \\
& =\frac{1}{\sqrt{2 \pi} \frac{D}{S} \frac{\sigma}{(\Delta t)^{1 / 2}}} \frac{e^{-\frac{1}{2} \frac{\left(\frac{x}{D / S}-1\right)^{2}}{\frac{\sigma^{2}}{4(\Delta t)}\left(\frac{x}{D / S}+1\right)^{2}}}}{\frac{1}{4}\left(\frac{x}{D / S}+1\right)^{2}} .
\end{aligned}
$$

Note that when $x \approx D / S$ the factor $\left(\frac{x}{D / S}+1\right)^{2} \approx 4$ cancels the $1 / 4$, so that $f_{X_{1}}(x)$ is approximately normal. However, we continue with the exact expression above, which can be expressed as

$$
f_{X_{1}}(x)=\frac{1}{\sqrt{2 \pi} \frac{D}{S} \frac{\sigma}{(\Delta t)^{1 / 2}}} \frac{e^{-\frac{1}{2} \frac{(x-D / S)^{2}}{4(\Delta t)}(x+D / S)^{2}}}{\frac{1}{4}\left(\frac{S}{D}\right)^{2}(x+D / S)^{2}} .
$$

For $G: \mathbb{R}^{+} \rightarrow \mathbb{R}$ satisfying Condition $G_{A}$, the density, $f_{2}$, of $X_{2}:=G\left(X_{1}\right)$ is given by

$$
f_{2}(y)=\frac{f_{1}\left(G^{-1}(y)\right)}{G^{\prime}\left(G^{-1}(y)\right)}=\frac{f_{1}(x)}{G^{\prime}(x)}
$$

\footnotetext{
${ }^{3}$ See Tong, 1990 [33] for a comprehensive exposition of the properties of the normal distribution
} 
where we use the notation $y=G(x), G^{-1}(y)=x$. Substitution into (15) yields the exact expression

$$
f_{2}(y)=\frac{1}{\sqrt{2 \pi} \frac{S}{D} \frac{\sigma}{(\Delta t)^{1 / 2}} G^{\prime}\left(G^{-1}(y)\right)} \frac{e^{-\frac{1}{2} \frac{\left(G^{-1}(y)-D / S\right)^{2}}{4(\Delta t)}\left(G^{-1}(y)+D / S\right)^{2}}}{\frac{1}{4}\left(G^{-1}(y)+D / S\right)^{2}} .
$$

Finally, the density $f_{3}$ of $X_{3}:=X_{2} \Delta t$, the quantity governing price change in (13) is given by $f_{3}(y)=f_{2}\left(\frac{y}{\Delta t}\right) / \Delta t$, so substitution into (16) yields the exact density given by the following.

Proposition 2.1. Let $G: \mathbb{R}^{+} \rightarrow \mathbb{R}$ be a function satisfying Condition $G_{A}$ and $Y$ be defined as the standard normal, $\mathcal{N}(0,1)$. Then the density of the random variable that constitutes the right hand side of the price equation (13)

$$
G\left(\frac{D}{S} \frac{1+\frac{\sigma}{2(\Delta t)^{1 / 2}} Y}{1-\frac{\sigma}{2(\Delta t)^{1 / 2}} Y}\right) \Delta t
$$

is given by

$$
f_{3}(y)=\frac{1}{\sqrt{2 \pi} \frac{S}{D} \frac{\sigma \Delta t}{(\Delta t)^{1 / 2}} G^{\prime}\left(G^{-1}\left(\frac{y}{\Delta t}\right)\right)} \frac{e^{-\frac{1}{2} \frac{\left(G^{-1}\left(\frac{y}{\Delta t}\right)-D / S\right)^{2}}{\frac{\sigma^{2}}{4(\Delta t)}\left(G^{-1}\left(\frac{y}{\Delta t}\right)+D / S\right)^{2}}}}{\frac{1}{4}\left(G^{-1}\left(\frac{y}{\Delta t}\right)+D / S\right)^{2}} .
$$

3. Asymptotics of the density. The density (18) can be studied asymptotically for small variance, i.e., $\sigma^{2}<<1$. In this section, we obtain an exact expression that will be used subsequently for asymptotic analysis. Toward this end, we express the terms involving $G^{-1}$ in an exact Maclaurin-Taylor expansion with remainder with $\zeta_{1}$ and $\zeta_{2}$ as the usual intermediate values between $y / \Delta t-y_{0} / \Delta t$ and define $\eta_{1}$ by $G\left(\eta_{1}\right)=\zeta_{1}$ :

$$
\begin{aligned}
G^{-1}\left(\frac{y}{\Delta t}\right)-\frac{D}{S} & =G^{-1}\left(\frac{y}{\Delta t}\right)-G^{-1}\left(\frac{y_{0}}{\Delta t}\right) \\
& =\left(G^{-1}\right)^{\prime}\left(\zeta_{1}\right)\left(\frac{y}{\Delta t}-\frac{y_{0}}{\Delta t}\right) \\
& =\frac{1}{G^{\prime}\left(\eta_{1}\right)}(y-G(D / S) \Delta t) \frac{1}{\Delta t}
\end{aligned}
$$

so that one has also

$$
G^{-1}\left(\frac{y}{\Delta t}\right)+\frac{D}{S}=2 \frac{D}{S}+\frac{1}{G^{\prime}\left(\eta_{1}\right)}(y-G(D / S) \Delta t) \frac{1}{\Delta t} .
$$


For the numerator of the exponent we need the next term in the series expansion:

$$
\begin{aligned}
G^{-1}\left(\frac{y}{\Delta t}\right) & =G^{-1}\left(\frac{y_{0}}{\Delta t}\right)+\left(G^{-1}\right)^{\prime}\left(\frac{y_{0}}{\Delta t}\right)\left(\frac{y}{\Delta t}-\frac{y_{0}}{\Delta t}\right) \\
& +\frac{\left(G^{-1}\right)^{\prime \prime}\left(\zeta_{2}\right)}{2}\left(\frac{y}{\Delta t}-\frac{y_{0}}{\Delta t}\right)^{2}
\end{aligned}
$$

and rewriting, we have

$$
\begin{aligned}
G^{-1}\left(\frac{y}{\Delta t}\right) & =\frac{D}{S}+\frac{1}{G^{\prime}\left(\frac{D}{S}\right)}(y-G(D / S) \Delta t) \frac{1}{\Delta t} \\
& +\frac{\left(G^{-1}\right)^{\prime \prime}\left(\zeta_{2}\right)}{2} \frac{(y-G(D / S) \Delta t)^{2}}{(\Delta t)^{2}} .
\end{aligned}
$$

From Condition $G_{A}$, the first and second derivatives $G^{-1}$ are bounded. Writing

$$
\begin{gathered}
f_{3}=: e^{E} / B \\
E:=-\frac{1}{2} \frac{\left(G^{-1}\left(\frac{y}{\Delta t}\right)-G^{-1}\left(\frac{y_{0}}{\Delta t}\right)\right)^{2}}{\frac{\sigma^{2}}{\Delta} \frac{1}{4}\left(G^{-1}\left(\frac{y}{\Delta t}\right)+G^{-1}\left(\frac{y_{0}}{\Delta t}\right)\right)^{2}}
\end{gathered}
$$

we examine the terms $E$ and $B$ below, so that substitution of the expansions (19), (20) in numerator and denominator yields

$$
E=-\frac{1}{2} \frac{\left(\frac{1}{G^{\prime}\left(\frac{D}{S}\right)}(y-G(D / S) \Delta t) \frac{1}{\Delta t}+\frac{\left(G^{-1}\right)^{\prime \prime}\left(\zeta_{2}\right)}{2} \frac{(y-G(D / S) \Delta t)^{2}}{(\Delta t)^{2}}\right)^{2}}{\frac{\sigma^{2}}{\Delta t} \frac{1}{4}\left(2 \frac{D}{S}+\frac{1}{G^{\prime}\left(\eta_{1}\right)}(y-G(D / S) \Delta t) \frac{1}{\Delta t}\right)^{2}} .
$$

Factoring the key terms, one has

$$
E=-\frac{1}{2} \frac{\left((y-G(D / S) \Delta t)+\frac{G^{\prime}\left(\frac{D}{S}\right)\left(G^{-1}\right)^{\prime \prime}\left(\zeta_{2}\right)}{2} \frac{(y-G(D / S) \Delta t)^{2}}{(\Delta t)}\right)^{2}}{\left[G^{\prime}\left(\frac{D}{S}\right)\right]^{2} \sigma^{2} \Delta t\left(\frac{D}{S}\right)^{2}\left(1+\frac{1}{2 G^{\prime}\left(\eta_{1}\right) D / S}(y-G(D / S) \Delta t) \frac{1}{\Delta t}\right)^{2}} .
$$

We will analyze this expression in the next section to show that (for fixed $\Delta t$ ) the terms beyond leading order can be controlled. Next we manipulate the expression for $B$, the denominator of $f_{3}$ given by (18) :

$$
\begin{aligned}
B & :=\sqrt{2 \pi} \frac{S}{D} \frac{\sigma \Delta t}{(\Delta t)^{1 / 2}} G^{\prime}\left(G^{-1}\left(\frac{y}{\Delta t}\right)\right) \frac{1}{4}\left(G^{-1}\left(\frac{y}{\Delta t}\right)+D / S\right)^{2} \\
& =\sqrt{2 \pi} \frac{S}{D} \sigma(\Delta t)^{1 / 2} G^{\prime}\left(G^{-1}\left(\frac{y}{\Delta t}\right)\right)\left(\frac{D}{S}\right)^{2} . \\
& \left(1+\frac{1}{2 \frac{D}{S} G^{\prime}\left(\eta_{1}\right)}(y-G(D / S) \Delta t) \frac{1}{\Delta t}\right)^{2} .
\end{aligned}
$$


Using the series expansion for $G^{-1}\left(\frac{y}{\Delta t}\right)$, namely, (19) together with the expansion for $G^{\prime}$ we can write the Maclaurin-Taylor expression,

$$
\begin{aligned}
G^{\prime}\left(G^{-1}\left(\frac{y}{\Delta t}\right)\right) & =G^{\prime}\left(\frac{D}{S}+\frac{1}{G^{\prime}\left(\eta_{1}\right)}(y-G(D / S) \Delta t) \frac{1}{\Delta t}\right) \\
& =G^{\prime}\left(\frac{D}{S}\right)+G^{\prime \prime}(\xi)\left(\frac{1}{G^{\prime}\left(\eta_{1}\right)}(y-G(D / S) \Delta t) \frac{1}{\Delta t}\right)
\end{aligned}
$$

in terms of another intermediate value $\xi$ between $y / \Delta t$ and $y_{0} / \Delta t$. Substitution into (23) and some algebraic simplification yields

$$
\begin{aligned}
B & =\sqrt{2 \pi} \frac{D}{S} \sigma(\Delta t)^{1 / 2}\left\{G^{\prime}\left(\frac{D}{S}\right)+G^{\prime \prime}(\xi)\left(\frac{1}{G^{\prime}\left(\eta_{1}\right)}(y-G(D / S) \Delta t) \frac{1}{\Delta t}\right)\right\} \\
& \cdot\left(1+\frac{1}{2 \frac{D}{S} G^{\prime}\left(\eta_{1}\right)}(y-G(D / S) \Delta t) \frac{1}{\Delta t}\right)^{2} \cdot
\end{aligned}
$$

Having used the Taylor expansions to rewrite $E$ and $B$, we have an exact form of $f_{3}=: e^{E} / B$ that is the density for the variable expressed in (17). We will analyze these terms further in the next section.

4. Analysis of the limits. Our next objective is to analyze the density, $f_{3}$, obtained above for the random variable, $G(\ldots) \Delta t$, in (11), that is an expression of the density of relative price changes, through (10), in a time interval $(t, t+\Delta t)$.

One implication of this analysis will be to establish rigorously the power-law tail of the density of relative price change. We will also compare the density $f_{3}$ for $X_{3}$ (given by (18)) with the normal approximation denoted by $X_{3}^{(N)}$ and defined below in (26). One feature of the quotient of normals that has long been established is that its density near the mean is close to a normal under a broad set of assumptions on the parameters. We will see in Section 5 that an implication of this idea can be used to derive stochastic differential equations for relative price change.

A tool we will use below is the basic result in asymptotic Laplace integrals for a continuous, bounded function $S$ and a twice continuously differentiable function $h$ with maximum at 0 . Assuming $a>>1$, one has

$$
I:=\int_{-\infty}^{\infty} u(x) e^{a h(x)} d x=u(0)\left(\frac{-2 \pi}{a h^{\prime \prime}(0)}\right)^{1 / 2} e^{a h(0)}+e^{a h(0)} O\left(a^{-3 / 2}\right)
$$

(see e.g., Murray, 2012 [24] p. 34, and the classic text by De Bruijn, 1981 [11]). Our goal is to prove that the expectation of an arbitrary bounded, continuous function $R$ with respect to $X_{3}$ is given by the expectation of $R$ with respect to

$$
X_{3}^{(N)} \sim \mathcal{N}\left(G(D / S) \Delta t, \sigma^{2} \Delta t\left(G^{\prime}\left(\frac{D}{S}\right) \frac{D}{S}\right)^{2}\right)
$$


plus terms of order $\sigma^{2}$.

Theorem 4.1. Let $f_{3}$ and $f_{3}^{(N)}$ be defined as the densities of $X_{3}$ and $X_{3}^{(N)}$, defined by (18) and (26). For fixed $\Delta t$ and an arbitrary bounded, continuous function $R$, one has

$$
\left|\int_{-\infty}^{\infty} R(x) f_{3}(x) d x-\int_{-\infty}^{\infty} R(x) f_{3}^{(N)}(x) d x\right| \leq C \sigma^{2}
$$

where $C$ is a constant that is independent of $\sigma^{2}$.

Remark. Inequality (27) implies that $X_{3}$ converges as $\sigma \rightarrow 0$ to $X_{3}^{(N)}$ in distribution (see Appendix D).

Proof. (i) First we consider the limiting integral

$$
I_{1}=\int_{-\infty}^{\infty} R(y) f_{3}^{(N)}(y) d y
$$

We have then, with $z:=y-G(D / S) \Delta t$ and $\tilde{R}(z)=\tilde{R}(y-G(D / S) \Delta t)=$ $R(y)$, the expressions

$$
\begin{aligned}
I_{1} & =\frac{1}{\sigma(\Delta t)^{1 / 2}} \int_{-\infty}^{\infty} R(y) \frac{e^{-\frac{1}{2} \frac{(y-G(D / S) \Delta t)^{2}}{\sigma^{2} \Delta t\left(G^{\prime}\left(\frac{D}{S}\right) \frac{D}{S}\right)^{2}}}}{\sqrt{2 \pi} G^{\prime}\left(\frac{D}{S}\right) \frac{D}{S}} d y \\
& =\frac{1}{\sigma(\Delta t)^{1 / 2}} \int_{-\infty}^{\infty} \tilde{R}(z) \frac{e^{-\frac{1}{2} \frac{z^{2}}{\sigma^{2} \Delta t\left(G^{\prime}\left(\frac{D}{S}\right) \frac{D}{S}\right)^{2}}}}{\sqrt{2 \pi} G^{\prime}\left(\frac{D}{S}\right) \frac{D}{S}} d z .
\end{aligned}
$$

Let $a:=\frac{1}{\sigma^{2} \Delta t}$ so $a>>1$, and

$$
\begin{aligned}
u(z) & :=\frac{\tilde{R}(z)}{\sqrt{2 \pi} G^{\prime}\left(\frac{D}{S}\right) \frac{D}{S}} \\
h(z) & :=-\frac{1}{2} \frac{z^{2}}{\left(G^{\prime}\left(\frac{D}{S}\right) \frac{D}{S}\right)^{2}},
\end{aligned}
$$

so differentiating twice leads to

$$
h^{\prime \prime}(0)=-\frac{1}{\left(G^{\prime}\left(\frac{D}{S}\right) \frac{D}{S}\right)^{2}} .
$$

Substitution into (25) yields

$$
\begin{aligned}
I_{1} & =a^{1 / 2} u(0)\left(\frac{-2 \pi}{a h^{\prime \prime}(0)}\right)^{1 / 2}+O\left(a^{-3 / 2}\right) \\
& =\tilde{R}(0) \frac{1}{\sqrt{2 \pi} G^{\prime}\left(\frac{D}{S}\right) \frac{D}{S}} \sqrt{2 \pi}\left(G^{\prime}\left(\frac{D}{S}\right) \frac{D}{S}\right)^{-1}+O\left(\sigma^{2} \Delta t\right) \\
& =R(D / S)+O\left(\sigma^{2} \Delta t\right) .
\end{aligned}
$$


(ii) Next, using the notation $\hat{R}(z):=R(y)$, consider the integral

$$
\begin{aligned}
I_{2} & =\int_{-\infty}^{\infty} R(y) f_{3}(y) d y \\
& =\int_{-\infty}^{\infty} \hat{R}(z) \frac{e^{a h_{1}(z)}}{B_{1}} d z
\end{aligned}
$$

where $h_{1}, B_{1}$ and $u_{1}$ are defined by

$$
\begin{gathered}
h_{1}(z):=-\frac{1}{2} \frac{\left(z+\frac{G^{\prime}\left(\frac{D}{S}\right)\left(G^{-1}\right)^{\prime \prime}\left(\zeta_{2}\right)}{2} \frac{z^{2}}{(\Delta t)}\right)^{2}}{\left[G^{\prime}\left(\frac{D}{S}\right) \frac{D}{S}\right]^{2}\left(1+\frac{1}{2 G^{\prime}\left(\eta_{1}\right) D / S} z \frac{1}{\Delta t}\right)^{2}} \\
B_{1}(z):=\sqrt{2 \pi} G^{\prime}\left(G^{-1}\left(\frac{y}{\Delta t}\right)\right) \frac{D}{S}\left(1+\frac{1}{2 \frac{D}{S} G^{\prime}\left(\eta_{1}\right)} \frac{z}{\Delta t}\right)^{2} \\
u_{1}(z):=\frac{\hat{R}(z)}{B_{1}(z)} .
\end{gathered}
$$

Note that $B_{1}(0)=\sqrt{2 \pi} \frac{D}{S} G^{\prime}\left(\frac{D}{S}\right)$ and $h_{1}^{\prime \prime}(0)=-\frac{1}{\left[\frac{D}{S} G^{\prime}\left(\frac{D}{S}\right)\right]^{2}}$ (see Appendix B). Note also that $\Delta t$ is a fixed constant. One has then

$$
\begin{aligned}
I_{2} & =a^{1 / 2} g(0)\left(\frac{-2 \pi}{a h_{1}^{\prime \prime}(0)}\right)^{1 / 2}+O\left(a^{-3 / 2}\right) \\
& =R(D / S)+O\left(\sigma^{2} \Delta t\right) .
\end{aligned}
$$

Since $I_{1}$ and $I_{2}$ differ by no more than a constant times $\sigma^{2}$ the inequality (27) is thus established, so expectations with respect to $X_{3}$ and $X_{3}^{(N)}$ are within $O\left(\sigma^{2}\right) \cdot / / /$

Remark. This result tells us that with $\Delta t>0$ fixed, the appropriately scaled normal density is an approximation to the density of $G \Delta t$. This is despite the fact that the densities are quite different at large values of the argument (i.e., exponential versus power law, or fat tail, decay). As an alternative, one can choose the small parameter as $\sigma(\Delta t)^{-1 / 2}$ and allow $\Delta t$ to approach zero as well, so long as $\sigma(\Delta t)^{-1 / 2} \rightarrow 0$. This is discussed in Appendix C.

\section{The stochastic dynamics equation through the supply/demand} equation.

5.1. Volatility. In this section we utilize the rigorous results obtained thus far to write the expression for the stochastic process for relative price change 
as well as expressions for volatility. Summarizing the results of the previous section, we have

$$
\begin{gathered}
P^{-1} \frac{\Delta P}{\Delta t}=X_{2}, \text { i.e., } P^{-1} \Delta P=X_{3} \\
X_{3}=X_{3}^{(N)}+O\left(\sigma^{2}\right)
\end{gathered}
$$

where $X_{3}^{(N)}$ is normally distributed [see (26) for definition]. Thus, the relative price change within the time interval $(t, t+\Delta t)$ is close to a normal for small variance, $\sigma^{2}$, and attains the normal limit (in the sense of distributions) as $\sigma \rightarrow 0$. Together these yield the expression (to leading order in $\sigma$ )

$$
P^{-1} \Delta P \sim \mathcal{N}\left[G\left(\frac{D}{S}\right) \Delta t, \sigma^{2}(\Delta t)\left\{\frac{D}{S} G^{\prime}\left(\frac{D}{S}\right)\right\}^{2}\right] .
$$

which can also be expressed as

$$
P^{-1} \Delta P=G(D / S) \Delta t+\sigma G^{\prime}(D / S)(D / S) \Delta W
$$

where $\Delta W$ is normal with mean zero and variance $\Delta t$.

Using the basic theory (see e.g., Schuss, 2009 [29]) we set $[W(t) ; t \geq 0]$ as the stochastic process defined on the probability space $(\Omega, \mathcal{F}, \mathbb{P})$ that satisfies the conditions of Brownian motion. In particular, $\mathbb{P}\{W(0)=0\}=1$, the increments $\Delta W:=W(t+\Delta t)-W(t)$ are independent of events prior to $t$ and $\Delta W \sim \mathcal{N}(0, \Delta t)$. As a function of $t, W$ is continuous except possibly on a set of measure zero in $\Omega$.

With this formalism, we can write the stochastic process for $P$, based on (29), in the limiting form as

$$
d P=G(D / S) P d t+\sigma G^{\prime}(D / S)(D / S) P d W
$$

Ito's formula applied to the function $\log P$ then yields

$$
d \log P=\left\{G(D / S)-\frac{1}{2}\left(\sigma G^{\prime}(D / S)(D / S)\right)^{2}\right\} d t+\sigma G^{\prime}(D / S) D / S d W
$$

Thus, taking the difference between (30) and (31) yields

$$
d \log P=\frac{1}{P} d P+O\left[\sigma^{2}\right]
$$

For small $\sigma$, one can formally neglect the $O\left[\sigma^{2}\right]$ terms, and the solutions of the two stochastic equations (30) and (31) can be expected to be close in the appropriate norm. However, we utilize the exact formulation (30).

Note that equation (30) can be written, using Condition $G$ in a form that explicitly displays the symmetry between supply and demand, as

$$
d P=\frac{1}{2}\{G(D / S)-G(S / D)\} P d t+\sigma\left\{G^{\prime}(D / S)(D / S)-G^{\prime}(S / D)(S / D)\right\} P d W
$$


The marginal volatility of the price, $P$, is then defined and calculated (see Appendix E) as ,

$$
\mathbb{V}_{P}(t):=\lim _{\Delta t \rightarrow 0} \frac{\operatorname{Var}[\Delta P(t)]}{\Delta t}=E\left[\left(\sigma P G^{\prime}(D / S) D / S\right)^{2}\right],
$$

where $\Delta P(t):=P(t+\Delta t)-P(t)$. We can also write this expression as

$$
\mathbb{V}_{\frac{P}{E[P]}}(t):=\lim _{\Delta t \rightarrow 0} \frac{1}{\Delta t} \operatorname{Var}\left[\frac{\Delta P(t)}{E[P(t)]}\right]=E\left[\left(\frac{\sigma P G^{\prime}(D / S) D / S}{E[P]}\right)^{2}\right] .
$$

Within our formalism, this is the formula that most closely expresses the marginal volatility of the relative price change. The analogous marginal volatility for $\log P$ is given by

$$
\mathbb{V}_{\log P}(t):=\lim _{\Delta t \rightarrow 0} \frac{\operatorname{Var}[\Delta \log P(t)]}{\Delta t}=E\left[\left(\sigma G^{\prime}(D / S) D / S\right)^{2}\right] .
$$

Note that $D$ and $S$ can also be considered as functions of $P$ in which case (30) becomes a more complicated stochastic differential equation.

5.2. Implications of the supply/demand based stochastic asset dynamics equation. The equation (30) has been derived from supply/demand considerations and the resulting mathematics of a ratio of normals, while the classical asset price dynamics equation (4) is based on an idealization of empirical observations. The equation (4) is built on the assumption that the price change and volatility are independent, while (30) shows that if one considers a supply/demand model that is close in principle to classical economics, then one is forced into the conclusion that volatility and price change are closely connected. In particular, (35) has the consequence that when relative prices are changing most rapidly, the volatility is highest, while it will be lowest at market extrema. This is consistent with an empirical study of 40 major market tops and subsequent declines, in which Sornette et. al., 2018 31] showed that for two-thirds of the cases, volatility is lowest as the market peaks and higher as the market rises or falls rapidly. Similarly, Bitcoin exhibits a valley in volatility near its peak trading price in December 2017 (Caginalp and Caginalp, 2018 [9]).

A key idea in this approach is that the stochastics in prices arises from random changes in supply and demand near the trading price. In other words, if one knew how the supply and demand would change, then one could determine how market makers and arbitrageurs would react, and have an accurate gauge for price formation.

The approach that leads to (30) has a natural consequence in that volatility will be greatest when the supply/demand imbalance is greatest, which is also when the magnitude of the relative price change is largest. One caveat is that momentum trading, which is not part of this model, could alter this conclusion. Risk assessment is one of the areas in which the practical applications of utilizing (30) can lead to better results. Options pricing, e.g., the Black-Scholes equation, is another. 
The analysis we have presented involves only the mathematics of supply and demand. During market tops and bottoms, there are a number of additional issues. Market bottoms, in particular, feature numerous additional motivations that are both classical and behavioral. For example, during market bottoms, one has margin calls, whereby investors who have bought stock partially with borrowed money are required to sell some stock unless they are able to provide more cash. In addition there are strategies whereby investors seek to balance portfolios which require selling as stocks are nearing a bottom. Behavioral factors such as risk aversion and fear can also play a part in adding additional volatility near market bottoms. These additional factors can be built on the general supply/demand model we have presented. In particular, the expected supply and demand can be determined by differential equations that are coupled to the price equation (30). With the inclusion of trend based motivations, there is likely to be a more complex interaction between market extrema and volatility.

Among the practical implications of this analysis is that in measuring volatility one should select a broad time frame that includes rising, falling, peaking and bottoming phases of a market in calculating volatility. A refinement of this concept can be attained by obtaining correlations of volatility on various time intervals with the price trend on those intervals.

Ultimately, this approach highlights the need for delving deeper into the motivations of traders and investors so that expected returns and volatility can be analyzed more comprehensively through data.

Throughout this discussion we are assuming that $D(t)$ and $S(t)$ are given functions. The analysis is completely general and allows for any set of motivations or strategies that may determine $D(t)$ and $S(t)$. In particular, one can combine the analysis of this paper with the asset flow equations (see for example, [6]) in which supply and demand depend upon trend, valuation and the ratio of available cash to asset. The randomness in the supply and demand that we have considered in this paper can be incorporated within the price equation in that system of equations. Furthermore, the supply and demand can depend on other strategies and behavioral motivations.

5.3 Testing using models and empirical data. If supply and demand are described by differential equations utilizing microeconomic principles, then a numerical computation can be utilized to generate a set of prices whereby one can compute the marginal volatility defined above. In particular, let $\mathcal{A}:=$ $\left\{t_{1}, t_{2}, . . t_{M}\right\}$ be the grid for the numerical computation on the interval $[0, T]$ with uniform spacing $\delta t=t_{i}-t_{i-1}$. Given any $t_{i *} \in \mathcal{A}$, we consider the interval

$$
\left[t_{i *}, t_{i *}+\Delta t\right]=\left[t_{i *}, t_{i *+K}\right]
$$

i.e., the interval $\left[t_{i *}, t_{i *}+\Delta t\right]$ contains the $K$ points of the grid $\left\{t_{i *}, t_{i *+1}, \ldots t_{i *+K}\right\}$ and $K \delta t=\Delta t$.

Using the relations

$$
\Delta P=P\left(t_{i *}+\Delta t\right)-P\left(t_{i *}\right)=\sum_{j=1}^{K}\left\{P\left(t_{i *+j \delta t}\right)-P\left(t_{i *+(j-1) \delta t}\right)\right\}
$$




$$
\begin{aligned}
\operatorname{Var}[\Delta P] & =\operatorname{Var}\left[\sum_{j=1}^{K}\left\{P\left(t_{i *+j}\right)-P\left(t_{i *+(j-1)}\right)\right\}\right] \\
& \tilde{=} \operatorname{Var}\left[\left\{P\left(t_{i *+j}\right)-P\left(t_{i *+(j-1)}\right)\right\}\right],
\end{aligned}
$$

we treat $\left\{S_{j}\right\}=\left\{P\left(t_{i *+j}\right)-P\left(t_{i *+(j-1)}\right)\right\}$ as a set of random variables and compute the variance in the usual way, $\operatorname{Var}\left[\left\{S_{j}\right\}\right]=E\left[S_{j}^{2}\right]-\left(E\left[S_{j}\right]\right)^{2}$ and expectation, $E[P(t)]=K^{-1} \sum_{j=0}^{K-1} P\left(t_{i *+j}\right)$.

This can be used to compute the approximation to (35):

$$
\mathbb{V}_{\frac{P}{E[P]}}(t) \stackrel{1}{=} \frac{1}{\Delta t} \operatorname{Var}\left[\frac{\Delta P(t)}{E[P(t)]}\right]=\frac{\operatorname{Var}\left[\left\{S_{j}\right\}\right]}{\delta t} \frac{1}{E[P(t)]}
$$

and compared with the exact value.

Given market data in the same form, i.e., a set of values $\mathcal{A}:=\left\{t_{1}, t_{2}, . . t_{M}\right\}$ and an interval $(t, t+\Delta t)$ with prices $\left\{P\left(t_{i *}\right), P\left(t_{i *+1}\right), \ldots, P\left(t_{i *+K}\right)\right\}$ one can compute the marginal volatility above. Using this information one can test whether the peak of $\mathbb{V}_{P / E[P]}(t)$ occurs as prices change most rapidly, and whether the minimum occurs when prices are at an extremum. Empirical work by Sornette et. al., 2018 [31] provides some support for this theoretical conclusion in two-thirds of major market tops. Similarly, this can similarly be tested in mathematical and computer generated models.

Acknowledgements. The authors thank two anonymous referees for raising insightful questions and suggestions for improvement of the manuscript.

Conflict of Interest. The authors declare that they have no conflict of interest.

Appendix A. Discussion of market orders versus limit orders. In most markets such as the NYSE, there are several types of orders that can be placed. From the perspective of economics, the limit order is natural; the trader is willing to buy at any price below a set price, and analogously for sell orders. In a "market order" the trader accepts the current trading price, and makes the transaction immediately. A market order appears to be suboptimal from the perspective of basic economics, as the trader is accepting, without conditions, any prevailing price. Bae et. al., 2003 [2, however, present the following argument of the advantages of market orders when conditions are changing rapidly. "Consider a world where transaction prices move solely in response to information. In this world, the placement of limit orders is clearly unattractive because they have option features. A trader who submits a limit buy (sell) order provides the market with a free put (call) option. When the underlying value of the asset moves against the trader who submits a limit order, the limit order will be executed and the trader loses. When the value of an asset moves in favor of the trader, a limit order will never be executed." 
The traders' choice between market and limit orders has been studied in several papers. Keim and Madhaven 1998, 23]) provide evidence that traders such as indexers are likely to use market orders, however, traders who believe they have information on value tend to use limit orders. In data on 30 stocks from 1990 to 1991, Bae et. al., 2003 [2] found that 43.7\% of the trades were market orders. Harris and Hasbrouk, 1996 [19] found that 50\% of orders in their data set were market orders. A brokerage advertisement states: "The vast majority of market orders executed receive a price better than the nationally published quote." This suggests that the principal reason for placing a limit order, namely to obtain a better price, is eliminated. Also, brokers like market orders since they are always executed, thereby generating a commission, and possibly margin interest, unlike limit orders which are often not executed.

In addition to market and limit orders, there are several other types of orders, a frequently used one being "stop-loss," whereby an automatic market order is placed to sell if the trading price drops below a set level (and analogously for buy orders). These orders have higher priority than market orders, which have higher priority than limit orders at the same price. While the stop-loss seems antithetical to classical economics, it is widely used, as time is of essence in many cases.

The literature also notes that market orders tend to be preferred, as one would expect, when the bid/ask spread is small, trading is active, and volatility is not extremely high (e.g., Bae et. al., 2003 [2]). Thus, market orders can be assumed to be a substantial fraction of the total trades for stocks with large trading volume.

In determining price changes, the limit orders are less important for several reasons. First, if the limit price is far from the current trading price, the order does not have any impact on trading. Second, the limit orders have lower priority in execution, as noted above. Third, those who place limit orders generally operate on a longer time scale, so they are less concerned about short term price changes. For example, an investor who is influenced by value may feel that the price of $\$ 20$ for a stock may be a good buy, and would place an order that is valid for up to 60 days, with the hope that the stock will be bought at $\$ 20$ and will be trading higher in the years to come.

Consequently, in analyzing stock price dynamics, the market orders can be expected to be the primary factor. The dominance of market orders has the implication that in the classic method for examining price equilibrium, the intersection of supply and demand curves becomes degenerate. In the classical goods model, each consumer has a price below which he is willing to buy, and analogously for the seller. The underlying assumption for goods and services is that change is sufficiently gradual that as the supply and demand change, the trading price shifts to the new equilibrium. However, for stocks, the supply/demand picture is very heavily weighted near the current trading price, and prices adjust quickly to new orders so that much of the supply/demand curves are irrelevant. The supply curve (with price on the horizontal axis, and quantity on the vertical) can be regarded as a piecewise constant function with two strictly increasing segments, with the middle plateau representing a region 
near the trading price. Likewise, the demand curve would consist of two strictly decreasing segments with a level segment in between. The classical intersection is thus replaced by an interval of overlap between supply and demand. To illustrate how these curves would attain this shape, we consider the hypothetical start of trading, when one can imagine the classical supply and demand curves are strictly increasing and decreasing, respectively. Once trading starts, the buyers who had limit buy orders just below the trading price are willing to increase their bid slightly, as they fear losing out due to a possible increase in prices. Those who had planned on placing a buy order above the trading price also see that they can obtain a better price. Although they would be receiving the market price with the limit order, they can change to a market order to have higher priority in the trading and avoid missing the purchase. In this way the smooth intersecting curves evolve into the curves in which one has plateaus near the trading price.

Appendix B. Calculation of the second derivative. We first state a basic mathematical calculation. Suppose that $r(x)$ and $s(x)$ are twice continuously differentiable functions whose derivatives are bounded on bounded subsets of the domain. Then we have the computation

$$
\begin{gathered}
\frac{\partial^{2}}{\partial x^{2}} H(x):=-\frac{1}{2} \frac{\partial^{2}}{\partial x^{2}} \frac{\left(x+r(x) x^{2}\right)^{2}}{c(1+s(x) x)^{2}} \\
=-\frac{1}{c(x s(x)+1)^{2}}\left(2 x r(x)+x^{2} \frac{\partial r(x)}{\partial x}+1\right)^{2} \\
-\frac{3}{c} x^{2}\left(s(x)+x \frac{\partial s(x)}{\partial x}\right)^{2} \frac{(x r(x)+1)^{2}}{(x s(x)+1)^{4}} \\
-\frac{1}{c(x s(x)+1)^{2}}\left(x+x^{2} r(x)\right)\left(2 r(x)+4 x \frac{\partial r(x)}{\partial x}+x^{2} \frac{\partial^{2} r(x)}{\partial x \partial x}\right) \\
-\frac{1}{2 c} x^{2} \frac{(x r(x)+1)^{2}}{(x s(x)+1)^{3}}\left(-4 \frac{\partial s(x)}{\partial x}-2 x \frac{\partial^{2} s(x)}{\partial x \partial x}\right) \\
-\frac{1}{2 c(x s(x)+1)^{3}}\left(x+x^{2} r(x)\right)\left(-8 s(x)-8 x \frac{\partial s(x)}{\partial x}\right)\left(2 x r(x)+x^{2} \frac{\partial r(x)}{\partial x}+1\right) .
\end{gathered}
$$

Evaluating this expression at $x=0$ and using the boundedness properties of $r(x)$ and $s(x)$, one has then

$$
\frac{\partial^{2}}{\partial x^{2}} H(0)=-\frac{1}{c} .
$$


We apply this proposition to (28) with

$$
\begin{aligned}
& r(z):=\frac{1}{2 \Delta t} G^{\prime}\left(\frac{D}{S}\right)\left(G^{-1}\right)^{\prime \prime}\left(\zeta_{2}(z)\right), \quad c:=2\left(\frac{D}{S} G^{\prime}\left(\frac{D}{S}\right)\right)^{2} \\
& s(z):=\frac{1}{2 \frac{D}{S} G^{\prime}\left(\eta_{1}\right) \Delta t}=\frac{\left(G^{-1}\right)^{\prime}\left(\zeta_{1}(z)\right)}{2 \Delta t \frac{D}{S}} .
\end{aligned}
$$

Recall that, from the original definitions, $\zeta_{1}$ and $\zeta_{2}$ are functions of $z$, as they are values between $y / \Delta t-y_{0} / \Delta t$ while $z:=y-G(D / S) \Delta t$. Thus $y \rightarrow G(D / S) \Delta t$ as $z \rightarrow 0$. For example, since $y_{0} / \Delta t=G(D / S)$ as $z \rightarrow 0$, one has $\zeta_{1,2} \rightarrow$ $G(D / S) \Delta t$. Clearly, then, on a bounded interval containing $y_{0} / \Delta t$, the first four derivatives of $G^{-1}$ are bounded so that $r^{\prime \prime}(z)$ and $s^{\prime \prime}(z)$ are bounded. We have then

$$
\frac{d^{2} h(0)}{d z^{2}}=-\frac{1}{\left(\frac{D}{S} G^{\prime}\left(\frac{D}{S}\right)\right)^{2}} .
$$

Appendix C. An alternative scaling. Another way to choose the small parameter starts with the same expressions for $f_{3}$, namely (18), in terms of $E$ and $B$. Now using, instead of $z$, the variable $w:=(y-G(D / S) \Delta t) / \Delta t$ and defining $R(y)=: \hat{R}(w)$ leads to

$$
\begin{gathered}
I_{2}=\int_{-\infty}^{\infty} R(y) f_{3}(y) d y=\int_{-\infty}^{\infty} \hat{R}(w) \hat{f}_{3}(w) \Delta t d w \\
\hat{f}_{3}(w) \Delta t d w=\frac{\exp \left\{-\frac{1}{2} \frac{\left(\frac{1}{G^{\prime}\left(\frac{D}{S}\right)} w+\frac{\left(G^{-1}\right)^{\prime \prime}\left(\zeta_{2}\right)}{2} w^{2}\right)^{2}}{\frac{\sigma^{2}}{\Delta t} \frac{1}{4}\left(2 \frac{D}{S}+\frac{1}{G^{\prime}\left(\eta_{1}\right)} w\right)^{2}}\right\} d w}{\sqrt{2 \pi} \frac{\sigma}{(\Delta t)^{1 / 2}} G^{\prime}\left(G^{-1}\left(\frac{y}{\Delta t}\right)\right) \frac{D}{S}\left(1+\frac{1}{2 \frac{D}{S} G^{\prime}\left(\eta_{1}\right)} w\right)^{2}} .
\end{gathered}
$$

At this point the integral $I_{2}$ has the same form as the previous scaling. Upon defining $\alpha:=\Delta t / \sigma^{2}$,

$$
\begin{gathered}
h_{1}(w):=-\frac{1}{2} \frac{\left(\frac{1}{G^{\prime}\left(\frac{D}{S}\right)} w+\frac{\left(G^{-1}\right)^{\prime \prime}\left(\zeta_{2}\right)}{2} w^{2}\right)^{2}}{\frac{1}{4}\left(2 \frac{D}{S}+\frac{1}{G^{\prime}\left(\eta_{1}\right)} w\right)^{2}} \\
u_{2}(w):=\frac{\hat{R}(w)}{\sqrt{2 \pi} G^{\prime}\left(G^{-1}\left(\frac{y}{\Delta t}\right)\right) \frac{D}{S}\left(1+\frac{1}{2 \frac{D}{S} G^{\prime}\left(\eta_{1}\right)} w\right)^{2}}
\end{gathered}
$$

the integral can be expressed as

$$
I_{2}=\alpha^{1 / 2} \int_{-\infty}^{\infty} u_{2}(w) e^{\alpha h_{1}(w)} d w .
$$


The standard result above then yields

$$
\begin{aligned}
I_{2} & =\alpha^{1 / 2}\left\{u(0)\left(\frac{-2 \pi}{\alpha h_{1}^{\prime \prime}(0)}\right)^{1 / 2}+O\left(\alpha^{-3 / 2}\right)\right\} \\
& =\frac{\hat{R}(0)}{\sqrt{2 \pi} G^{\prime}(D / S) \frac{D}{S}} \frac{\sqrt{2 \pi}}{h_{1}^{\prime \prime}(0)}+O\left(\frac{\sigma^{2}}{\Delta t}\right) \\
& =R(D / S)+O\left(\frac{\sigma^{2}}{\Delta t}\right) .
\end{aligned}
$$

The same result holds for $I_{1}$ as we show below:

$$
\begin{gathered}
I_{2}=\int_{-\infty}^{\infty} R(y) f_{3}^{(N)}(y) d y=\int_{-\infty}^{\infty} \hat{R}(w) f_{3}^{(N)}(w) \Delta t d w \\
\hat{f}_{3}(w) \Delta t d w=\frac{\exp \left\{-\frac{1}{2} \frac{\left(\frac{1}{G^{\prime}\left(\frac{D}{S}\right)} w\right)^{2}}{\frac{\sigma^{2}}{\Delta t}\left(\frac{D}{S}\right)^{2}}\right\} d w}{\sqrt{2 \pi} \frac{\sigma}{(\Delta t)^{1 / 2}} G^{\prime}\left(\frac{D}{S}\right) \frac{D}{S}} .
\end{gathered}
$$

Defining

$$
h_{0}(w):=-\frac{1}{2} \frac{\left(\frac{1}{G^{\prime}\left(\frac{D}{S}\right)} w\right)^{2}}{\left(\frac{D}{S}\right)^{2}}
$$

so $h_{0}(0)=0$ and $h_{0}^{\prime \prime}(w)=-\frac{1}{\left(G^{\prime}\left(\frac{D}{S}\right) \frac{D}{S}\right)^{2}}$ and

$$
u_{0}(w):=\frac{\hat{R}(w)}{\sqrt{2 \pi} G^{\prime}\left(\frac{D}{S}\right) \frac{D}{S}}
$$

yields the calculation

$$
\begin{aligned}
I_{1} & =\alpha^{1 / 2}\left\{u_{0}(0)\left(\frac{-2 \pi}{\alpha h_{0}^{\prime \prime}(0)}\right)^{1 / 2}+O\left(\alpha^{-3 / 2}\right)\right\} \\
& =\alpha^{1 / 2}\left\{\frac{\hat{R}(0)}{\sqrt{2 \pi} G^{\prime}\left(\frac{D}{S}\right) \frac{D}{S}}\left(\frac{2 \pi\left(G^{\prime}\left(\frac{D}{S}\right) \frac{D}{S}\right)^{2}}{\alpha}\right)^{1 / 2}+O\left(\alpha^{-3 / 2}\right)\right\} \\
& =R\left(\frac{D}{S}\right)+O\left(\frac{\sigma^{2}}{\Delta t}\right) .
\end{aligned}
$$

Comparing (38) and (37), we see that the difference between $I_{1}$ and $I_{2}$ is at most $C \sigma^{2} / \Delta t$.

Appendix D. Theorems on convergence. After two definitions, we state a theorem from probability (see, e.g., Billingsley 2008 [3] p. 352) that is utilized in the derivation. 
Definition. Let $X_{n}$ and $X$ be random variables with distributions $F_{n}$ and $F$. We say that $F_{n}$ converges weakly to $F$ (denoted $F_{n} \Longrightarrow F$ ) if

$$
\lim _{n \rightarrow \infty} F_{n}(x)=F(x)
$$

for every point of continuity $x$. We say that $X_{n}$ converges in distribution, denoted $X_{n} \Longrightarrow X$, if $F_{n} \Longrightarrow F$.

Definition. If $\mu_{n}$ and $\mu$ are the probability measures corresponding to $F_{n}$ and $F$ then we write $\mu_{n} \Longrightarrow \mu$ if

$$
\lim _{n \rightarrow \infty} \mu_{n}(A)=\mu(A)
$$

for every set of the form $A:=(-\infty, x]$.

Theorem D.1. The following are equivalent:

(i) $\mu_{n} \Longrightarrow \mu$, (ii) $\lim _{n \rightarrow \infty} \mu_{n}(A)=\mu(A)$ for every $\mu$-continuity set A.

(iii) $\int g d \mu_{n} \rightarrow \int g d \mu$ for every bounded, continuous real function $g$.

Appendix E. Calculation of volatility. Let $\mathcal{F}_{t}$ be a Brownian filtration (see Schuss, 2009 [29] p. 66 ) so that $\mathcal{F}_{t}$ adapted stochastic processes $f(t ; \omega)$ are independent of increments of the Brownian motion, $W(t ; \omega)$, that are prior to $t$. Let $H_{2}[0, T]$ consist of $\mathcal{F}_{t}$ adapted stochastic processes $f(t ; \omega)$ on the interval $[0, T]$ such that

$$
\int_{0}^{T} E f^{2}(s ; \omega) d s<\infty .
$$

Now let $f(t ; \omega) g(t ; \omega)$ be stochastic processes in $H_{2}[0, T]$ such that for some positive constant $C$ one has

$$
\sup _{t \in[0, T]}\left\{E f^{2}(t ; \omega), E g^{2}(t ; \omega)\right\}<C .
$$

Also, assume that $f$ and $g$ are continuous in $t$ except possibly on a set of measure zero in $\Omega$. We now consider a general stochastic process $Z(t ; \omega)$ defined by

$$
d Z=f d t+g d W
$$

which is equivalent to writing (suppressing the $\omega$ variable)

$$
Z(t)-Z\left(t_{0}\right)=\int_{t_{0}}^{t} f(s) d s+\int_{t_{0}}^{t} g(s) d W(s) .
$$

Theorem E.1. If the stochastic processes $f$ and $g$ satisfy (39), $Z$ is a stochastic process defined by (41), and $\Delta Z(t):=Z(t+\Delta t)-Z(t)$, then

$\operatorname{Var}[\Delta Z]=\operatorname{Var}\left[\int_{t}^{t+\Delta t} f d s\right]+2 E\left[\int_{t}^{t+\Delta t} f d s \int_{t}^{t+\Delta t} g d W\right]+\int_{t}^{t+\Delta t} E g^{2} d s$ 
and the marginal volatility $\mathbb{V}(t)$ satisfies

$$
\mathbb{V}(t):=\lim _{\Delta t \rightarrow 0} \frac{\operatorname{Var}[\Delta Z(t)]}{\Delta t}=E g^{2}(t) .
$$

Proof. The first identity, (42), follows from squaring (41), and noting that

$$
E \int_{t}^{t+\Delta t} g(s) d W(s)=0
$$

from the assumption that $g$ is $\mathcal{F}_{t}$ adapted, and using a basic property of Ito integrals (see e.g., Schuss, 2009 [29], p. 68):

$$
E\left[\left(\int_{t}^{t+\Delta t} g d W\right)^{2}\right]=\int_{t}^{t+\Delta t} E g^{2} d s
$$

To obtain the second identity we establish the following two bounds using the Cauchy-Schwarz inequality for integrals, and then for expectations. Note that from (39) one has

$$
\begin{aligned}
& \left(\int_{t}^{t+\Delta t} f d s\right)^{2} \leq\left(\int_{t}^{t+\Delta t} 1^{2} d s\right)\left(\int_{t}^{t+\Delta t} f^{2} d s\right), \\
& E\left[\left(\int_{t}^{t+\Delta t} f d s\right)^{2}\right] \leq \Delta t \int_{t}^{t+\Delta t} E f^{2} d s \leq C(\Delta t)^{2}
\end{aligned}
$$

upon taking expectations. Now, using Cauchy-Schwarz for expectations on the middle term in (42) yields

$$
\begin{aligned}
\left|E\left[\int_{t}^{t+\Delta t} f d s \int_{t}^{t+\Delta t} g d W\right]\right| & \leq E\left[\left(\int_{t}^{t+\Delta t} f d s\right)^{2}\right]^{1 / 2} E\left[\left(\int_{t}^{t+\Delta t} g d W\right)^{2}\right]^{1 / 2} \\
& \leq C \Delta t\left(\int_{t}^{t+\Delta t} E g^{2} d s\right)^{1 / 2} \leq C^{2}(\Delta t)^{3 / 2} .
\end{aligned}
$$

Upon dividing by $\Delta t$ and taking the limit of (42) one has from (43)

$$
\mathbb{V}(t)=\lim _{\Delta t \rightarrow 0} \int_{t}^{t+\Delta t} E g^{2}(s ; \omega) d s=E g^{2}(t ; \omega)
$$

since $g$ and $g^{2}$ are continuous in $t$ except possibly on a set of measure zero in $\Omega$. Thus, $\left(E g^{2}\right)(t)$ is a continuous function of $t$. ///

If $f$ and $g$ are functions in (41) also depend on $Z$, i.e., $f(Z, t ; \omega)$, and initial conditions $Z(0):=Z_{0}$ are imposed, we have a stochastic differential equation (SDE). We say that $Z$ is a solution in the Ito sense if $Z(t ; \omega) \in H_{2}[0, T]$ for 
all $T>0$ and (41) holds for almost all $\omega \in \Omega$. Also $f$ and $g$ satisfy a uniform Lipschitz condition if

$$
|f(Z, t ; \omega)-f(Y, t ; \omega)|+|g(Z, t ; \omega)-g(Y, t ; \omega)| \leq K|Z-Y|
$$

for some $K>0$ and all $Y, Z \in \mathbb{R}$.

The stochastic differential equation (41) has a unique solution in the Ito sense if $f$ and $g$ satisfy (44) for all $t$ and for almost all $\omega \in \Omega$. The trajectories $Z(t ; \omega)$ are then continuous for almost all $\omega \in \Omega$ (see e.g., Schuss, 2009 [29], p. $94)$.

(i) Applying these results first to $Z:=\log P$ and $f:=G(D / S), g:=$ $\sigma G^{\prime}(D / S) D / S$, i.e., the $\mathrm{SDE}$

$$
d \log P=G(D / S) d t+\sigma G^{\prime}(D / S) D / S d W,
$$

one obtains from the theorem above, the result, with $\Delta \log P(t):=\log P(t+\Delta t)-$ $\log P(t)$,

$$
\mathbb{V}_{\log P}(t):=\lim _{\Delta t \rightarrow 0} \frac{\operatorname{Var}[\Delta \log P(t)]}{\Delta t}=E\left[\left(\sigma G^{\prime}(D / S) D / S\right)^{2}\right] .
$$

(ii) Next, applying these results to $Z:=P$ with $f:=P G(D / S), g:=$ $\sigma P G^{\prime}(D / S) D / S$, i.e., the $\mathrm{SDE}$

$$
d P=P G(D / S) d t+\sigma P G^{\prime}(D / S) D / S d W
$$

yields, with $\Delta P(t):=P(t+\Delta t)-P(t)$, the marginal volatility,

$$
\mathbb{V}_{P}(t):=\lim _{\Delta t \rightarrow 0} \frac{\operatorname{Var}[\Delta P(t)]}{\Delta t}=E\left[\left(\sigma P G^{\prime}(D / S) D / S\right)^{2}\right],
$$

which we can also write as

$$
\mathbb{V}_{\frac{P}{E[(t)]}}(t):=\lim _{\Delta t \rightarrow 0} \frac{1}{\Delta t} \operatorname{Var}\left[\frac{\Delta P(t)}{E[P(t)]}\right]=E\left[\left(\frac{\sigma P G^{\prime}(D / S) D / S}{E[P(t)]}\right)^{2}\right] .
$$

\section{References}

[1] Bachelier, L. 1900. Théorie de la spéculation. Ann. Sci. École Norm. Sup. $\mathbf{1 7}, 21-86$.

[2] Bae, K.H., Jang, H. and Park, K.S., 2003. Traders' choice between limit and market orders: evidence from NYSE stocks. Journal of Financial Markets, 6, $517-538$.

[3] Billingsley, P., 2008. Probability and measure. John Wiley \& Sons. 
[4] Bossaerts, P., Plott, C., 2004. Basic Principles of Asset Pricing Theory: Evidence from Large-Scale Experimental Financial Markets, Review of Finance, 8, 135-169,

[5] Black, F., Scholes, M., 1973. The pricing of options and corporate liabilities. J. Political Economy, 81, 637-654.

[6] Caginalp, G., Balevonich, D., 1999. Asset flow and momentum: Deterministic and stochastic equations. Phil. Trans. Royal Soc. A, 357, 2119-2113.

[7] Caginalp, C. and Caginalp, G., 2018. The quotient of normal random variables and application to asset price fat tails. Physica A, 499, 457-471.

[8] Caginalp, C. and Caginalp, G., 2019. Price equations with symmetric supply/demand; implications for fat tails. Economics Letters, 176, 79-82.

[9] Caginalp, C. and Caginalp, G., 2019. Stochastic asset price dynamics and volatility using a symmetric supply and demand price equation. Physica A, 523, $807-824$.

[10] Champagnat, N., Deaconu M., Lejay A., Navet N. and Boukherouaa S., 2013. An empirical analysis of heavy-tails behavior of financial data: The case for power laws. HAL archives-ouvertes.

[11] De Bruijn, N.G., 1981. Asymptotic methods in analysis. Courier Corporation.

[12] DeSantis, M. and Swigon, D., 2018. Slow-fast analysis of a multi-group asset flow model with implications for the dynamics of wealth. PloS one, 13(11), p.e0207764.

[13] Díaz-Francés, E. and Rubio, F. J., 2013. On the existence of a normal approximation to the distribution of the ratio of two independent normal random variables. Stat. Papers, 1-15.

[14] New York Times, 1929, "Fisher sees stocks permanently high" p.8, October $16,1929$.

[15] Franses, P.H. and McAleer, M., 2002. Financial volatility: An introduction. Journal of Applied Econometrics, 17, 419-424.

[16] Gjerstad, S. and Dickhaut, J., 1998. Price formation in double auctions. Games and economic behavior, 22, 1-29.

[17] Gjerstad, S., 2007. The competitive market paradox. Journal of Economic Dynamics and Control, 31, 1753-1780.

[18] Gjerstad, S., 2013. Price dynamics in an exchange economy. Economic Theory, 52, 461-500. 
[19] Harris, L. and Hasbrouck, J., 1996. Market vs. limit orders: the SuperDOT evidence on order submission strategy. Journal of Financial and Quantitative analysis, 31, 213-231.

[20] Hirshleifer, J., Glazer, A. and Hirshleifer, D., 2005. Price theory and applications: decisions, markets, and information. Cambridge University Press.

[21] Hull, J. and White, A., 1998. Incorporating volatility updating into the historical simulation method for value-at-risk. Journal of Risk, 1, 5-19.

[22] Karatzas, I., Shreve, S.E., 1998. Methods of mathematical finance (Vol. 39, pp. xvi+-407). New York: Springer.

[23] Keim, D.B. and Madhavan, A., 1998. The cost of institutional equity trades. Financial Analysts Journal, 54, 50-69.

[24] Murray, J.D., 2012. Asymptotic analysis. Springer Science \& Business Media.

[25] Merdan, H. and Alisen, M., 2011. A mathematical model for asset pricing. Applied Mathematics and Computation, 218, 1449-1456.

[26] Milgrom, P., 2017. Discovering Prices: Auction Design in Markets with Complex Constraints. Columbia University Press.

[27] Plott, C.R. and Pogorelskiy, K., 2017. Call market experiments: Efficiency and price discovery through multiple calls and emergent Newton adjustments. American Economic Journal: Microeconomics, 9, 1-41.

[28] Porter, D., Rassenti, S., Roopnarine, A. and Smith, V., 2003. Combinatorial auction design. Proceedings of the National Academy of Sciences, 100(19), 11153-11157.

[29] Schuss, Z., 2009. Theory and applications of stochastic processes: an analytical approach. Springer Science \& Business Media.

[30] Saunders, D., 2009. Pricing timer options under fast mean-reverting stochastic volatility. Canadian Applied Mathematics Quarterly, 17, 737753 .

[31] Sornette, D., Cauwels, P. and Smilyanov, G., 2018. Can we use volatility to diagnose financial bubbles? lessons from 40 historical bubbles. Quantitative Finance and Economics, 2, 486-594.

[32] Stojanovic, S.D., 2005. Risk premium and fair option prices under stochastic volatility: the HARA solution. Comptes Rendus Mathematique, 340(7), $551-556$.

[33] Tong, Y., 1990. The multivariate normal distribution, Springer-Verlag, New York. 
[34] Watson, D., Getz, M., 1981. Price theory and its uses. University Press of America, Lanham, MD.

[35] Weintraub, E., 1979. Microfoundations, The compatibility of microeconomics and macroeconomics, Cambridge University Press, Cambridge, UK.

[36] Wilmott, P., 2013. Paul Wilmott on quantitative finance, John Wiley \& Sons. 